\title{
High-Performance Multipath Routing Algorithm Using CPEGASIS Protocol in Wireless Sensor Cloud Environment
}

\author{
0. Pandithurai1, Dr. C. Sureshkumar² \\ ${ }^{1}$ Anna University of Technology, Comibatore, India \\ ${ }^{2}$ J.K.K. Nattraja College of Engineering and Technology, Komarapalayam, India \\ Email: pandics@gmail.com,ck_sureshkumar@yahoo.co.in
}

Received 8 May 2016; accepted 30 May 2016; published 24 August 2016

Copyright ( 2016 by authors and Scientific Research Publishing Inc.

This work is licensed under the Creative Commons Attribution International License (CC BY). http://creativecommons.org/licenses/by/4.0/

(c) (i) Open Access

\section{Abstract}

The current IT cloud computing is playing a vital role in most of the areas such as Education, Research, Health care, etc. The cloud computing technology involving in sensor networks embedded system and IOT (Internet of Things). At present scenario, the sensors collected the information from the particular environment, where the sensors are fixed and transfer the collected information to cloud storage, here the challenge is the data transmission i.e. data that traverse from sensor to cloud environment are the big issue and maximum number of data loss is very high especially in dynamic routing environment. If data loss is identified in any routing path then automatically the information will transfer to alternate routing path. In this paper, we introduce a new algorithm for automatic routing path selection that can be integrated with cloud technology. This algorithm supports when data loss is found in the particular path of a network, then it selects an alternate route to transfer the data. The proposed model is comparatively more efficient than the prior methodologies. The implementation of the proposed work is done on NS3 simulator, and the performance metric is analyzed.

\section{Keywords}

Wireless Sensor, Cloud Computing, Internet of Things

\section{Introduction}

Wireless sensor networks are like a self organizing communication model, where all the sensors connect through wireless communication network channels in multi-hub environment [1]. Here the big challenge is to integrate 
sensors through internet and another challenge is to communicate the information through internet. The advantage of existing model is that the user can access the data at any time and any location [2] [3]. In such a case, the assigning of the address to each and every sensor node is very difficult and lots of sensor nodes present in the WSN lead to the storage problem. The data storage and process are moved to the cloud computing environment to rectify this problem [5]. Cloud computing is one of the most emerging technologies in the current scenario; the main characteristics of cloud are on-demand, resource polling, elasticity and broad network access [4]. The major concept of cloud is based on virtualization (like logical instances), so we can connect our physical sensors to logical virtual sensors.

During the construction of sensor networks, we are proposing CPEGASIS (Cluster Power-efficient gathering in Sensor Information system) protocol, for enabling multipath routing. This is based on clustering method and the gateway cluster supports to forward the user request to neighbor cluster where the Cluster head $(\mathrm{CH})$ checks whether the data present itself or not. If data present it has to forward the data to gateway otherwise the request is forwarded to neighbor cluster. The working model of CPEGASIS protocol is shown in Figure 1.

The architecture is normally explained about working procedure of WSN in cloud computing infrastructure. Cloud is act between user and WSN client. The client seeks data from the cloud based on user's request, and the cloud gets the information from WSN. WSN consists of physical sensor nodes which are constructed based on the application requirements. WSN senses the data and sends the relevant information to cloud and finally cloud is providing the service to user. Here, the problem arises, when the WSN sends the information through the network via multiple paths. Here data communication is processed all the possible network routes even network traffic and route failure possibly occur in the internet. So, in this paper, we try to find out failure path and transfer the packets at the instance of alternate route in the network.

\section{Related Works}

\subsection{Sensor Networks}

The architectural design of sensor network is consist of autonomous sensors where the sensors are used to monitor the physical environment of the sensor field. Each and every sensor nodes in a WSN are typically designed with radio transceiver, mini microcontroller, energy source and battery [6] [7]. The size and cost of the sensor nodes are based on characteristics, design, energy, memory and bandwidth especially the battery is to be defined the lifetime of sensor and sensor networks. The performance of wireless sensor networks is depending on three different technical areas such as sensing, communication and computation of the network [8]. In this sensor networks routing is challenge task, where the purpose of routing algorithm is transfer the data source to destination

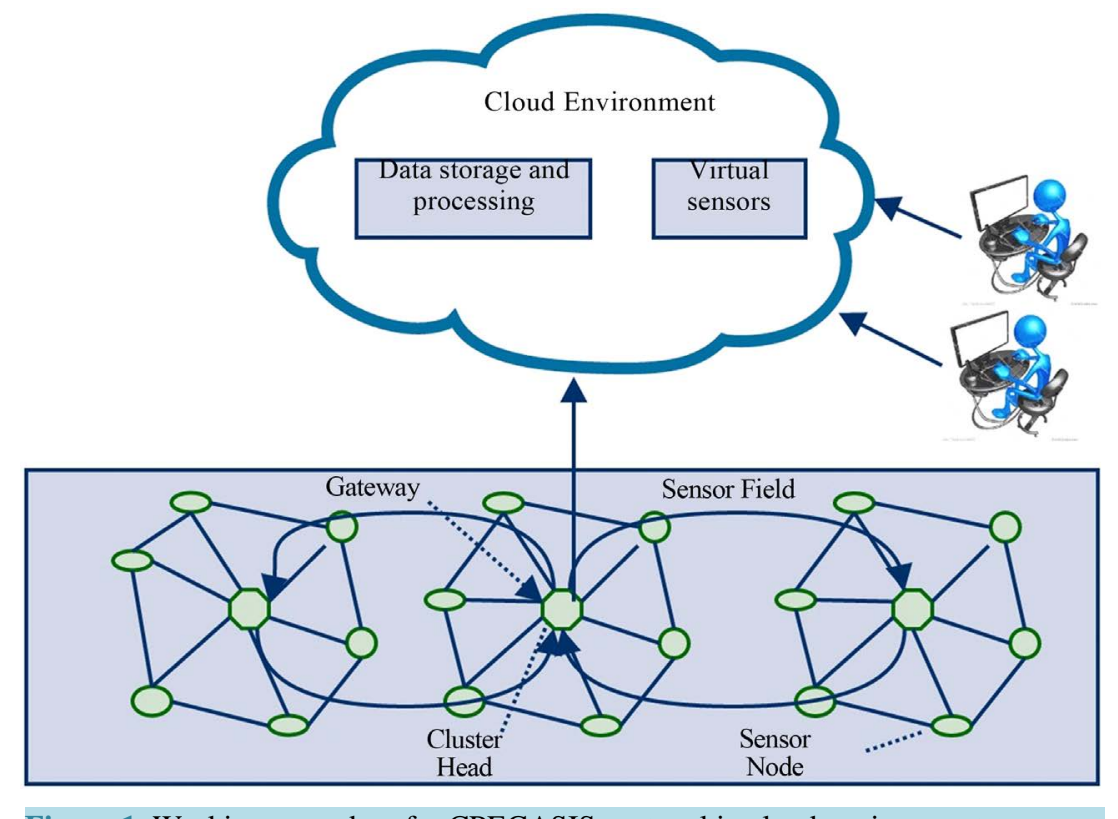

Figure 1. Working procedure for CPEGASIS protocol in cloud environment. 
with minimum distance in a network. Routing algorithms classified into three types such as is multipath routing, dynamic routing and hierarchical routing. The characteristics of these routing algorithms are based on protocol design, routing speed, type of routing, network topology and bandwidth [9].

\subsection{Routing Protocols in WSN}

Routing algorithm and protocol design are based on major two major categories one is based on Network structure and second category is based on protocol operation. Construction of network structure (based on protocol) is designed based on three methods includes flat based routing algorithm, hierarchical based routing algorithm and location based routing algorithm [10]. In Flat based routing method, the sensors are sense the data equally in WSN and also each and every sensor play a same role [11]. The second method is hierarchical based routing or cluster based routing, where the nodes are selected their own cluster head $(\mathrm{CH})$ and the cluster head selection is based on high energy and power. Cluster Head Gateway (CHG) receives the query from Base station (BS) and collect the relevant information from cluster heads and transfers the data to BS. The selection of cluster head is based on the high energetic power. That is, the most energetic cluster can act as cluster head of a gateway. The third method is location based routing algorithm where the method is based on the sensor field and incoming signal from the BS. Depending on the signal, the nearest sensor node to the BS communicates the information to BS [12].

The fourth method in WSN routing protocol is based on protocol operation, the protocol operation is working on five methods includes Multipath, Query, Quality of service, Coherent and Negotiation. Multipath enables communication between the nodes and the network. It is an alternative method for a single path communication [6]. Query process is based on user query, made in the network the sensor nodes are checking the relevant data itself and transfer the data depends on the query. Queries based on either natural language or high level language. The third important parameter is QOS; it is based on energy consumption and quality of the data in a WSN [13]. In coherent operation, the data if transfer to aggregators after a process. The process is collection of task; it includes time and also duplicate prevention. Negotiation operation is totally work to avoid redundant data transmission in WSN.

\section{Proposed System}

The proposed methodology is enhancement of our existing Wireless sensor networks in Cloud environment (WSCE) using CPEGASIS (Cluster Power-efficient gathering in Sensor Information system) protocol. That particular model is based on data gathering based on CPEGASIS protocol this CPEGASIS protocol is an advancement of LEACH (Low-energy adaptive clustering hierarchy) and PEGASIS (Power-efficient gathering in Sensor Information system). In this working model the major challenge is data transmission without delay and increase the performance of data communication from WSN. In this paper we introduce a new routing algorithm that is High Performance Adaptive Multipath Routing (HPAMR) the process of HPAMR is explained in section 3.1. The HPAMR is working architecture shown in Figure 2.

\subsection{Clustering in WSN}

Clustering technique is using in the proposed in WSCE and the section of cluster head is based on energy. Energy calculation is based on supply of voltage. That is calculated depended on communication, data transmission and every individual current. All the nodes have data and nodes controlled by cluster head. Gateway $\mathrm{CH}$ has all information about CH. The address format of the Gateway Cluster Head is defined in the following address format.

\begin{tabular}{llll}
\hline Cluster ID & No of nodes in Cluster & Energy level & Data \\
\hline
\end{tabular}

Based on this address format each and every cluster has this information in the WSN. Energy level we discussed already and Data is defining here the current state of the data.

\subsection{High Performance Adaptive Multipath Routing}

The proposed High Performance Adaptive Multipath Routing algorithm (HPAMR) is used with CPEGASIS protocol in the proposed system. The working process of HPAMR algorithm multipath is selected and resource 


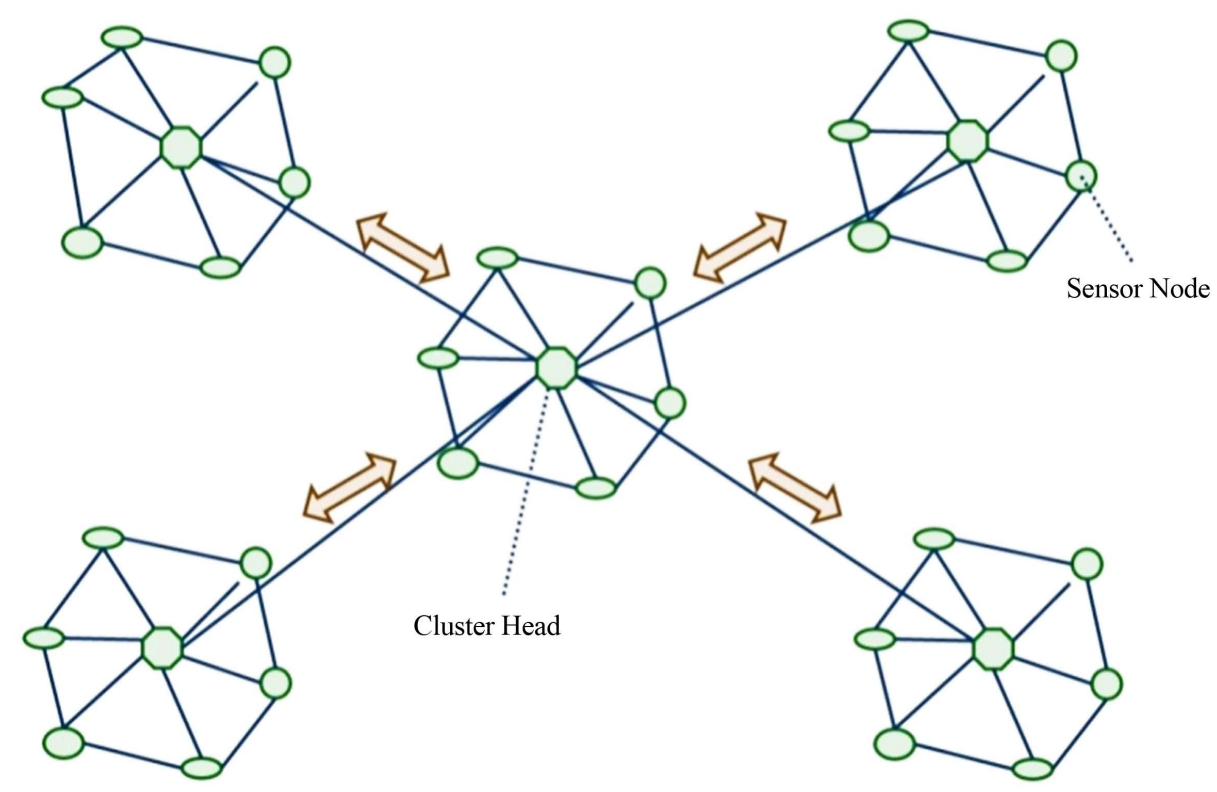

Figure 2. High performance adaptive multipath routing architecture.

equally distributed in the Wireless sensor networks, Here all the network path may not have a equal capacity so load is equally distributed and transfer the data in the network if congestion is occur in the network then verify the status of neighbors. If any neighbor is free then the resource is forwarded to that neighbor cluster head. These processes are avoiding unwanted retransmission process from the sender $\mathrm{CH}$ and reduce the data packet transmission delay and using this HPAMR algorithm we reduce the transmission delay and increase the transmission speed in WSN. In a single path routing algorithm the data process based on source and destination if the path is failure over all process is fail. So we are using multi path routing in this method if any failure occur in the network we need to retransmit the data from sender so in the proposed method we are using High Performance Adaptive Multipath Routing algorithm it identify the route failure and check the nearest neighbors $\mathrm{CH}$ whether the $\mathrm{CH}$ is free or not if the $\mathrm{CH}$ is free the current $\mathrm{CH}$ forward the data to that cluster head so here data delay is totally reduced and speed is increased. The proposed HPAMR working process is designed based on the following algorithm.

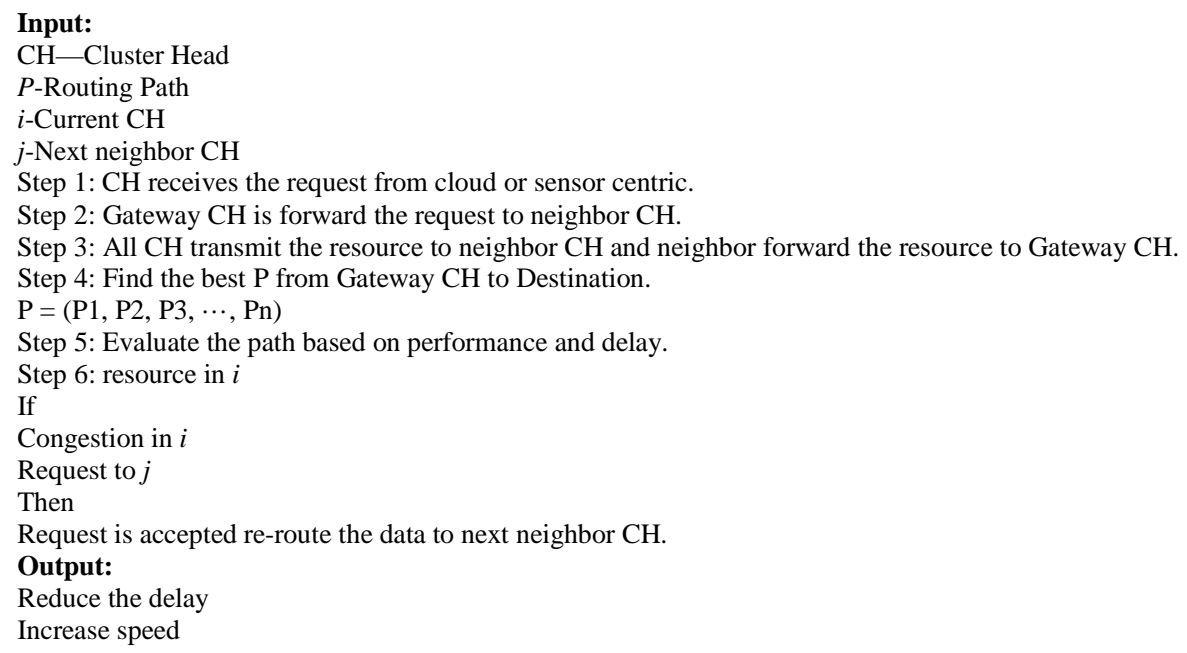

Finally based on proposed algorithm in a wireless sensor network we reduce the delay in network and increase the transmission speed. 


\section{Implementation}

The simulation of proposed working model designed using NS 3.23 version in Ubuntu 14.04. The simulation infrastructure designed for WSN, here all the nodes make a cluster and deployed in the field. The communication is based on wireless and data transmission is done gateway $\mathrm{CH}$ to destination. Finally the result of proposed HPAMR algorithm give a better result like reduce the delay of transmission and increase the transmission speed. We take the following parameter for simulation in Table 1.

\section{Result and Discussion}

The working model of proposed system we discussed already, the performance evaluation of proposed system is compare with Multipath routing algorithm. In wireless sensor networks based on the performance some of the algorithm maximum using in the real world namely dynamic routing, hierarchical routing and Multipath routing with these routing algorithm multipath routing algorithm is a best routing algorithm so we compared our proposed High Performance Adaptive Multipath Routing algorithm (HPAMR). In proposed model is comparatively high Fault tolerance and speed.

\subsection{Fault Tolerance}

The working process of HPAMR algorithm using CPEGASIS protocol in WSCE, the cluster head transfer the data in network if congestion occur in the wireless channels the cluster head the available neighbor cluster head status that is whether the $\mathrm{CH}$ is free or not. If the alternate cluster head free then the current $\mathrm{CH}$ is forward the packet to that $\mathrm{CH}$ so here we can totally avoid the packet loss and reduce the delay also. Comparatively the proposed HPAMR is best because here if the route failure or congestion occurs in network automatically the $\mathrm{CH}$ is forward to neighbor $\mathrm{CH}$. The performance comparison of existing multipath routing and proposed high performance adaptive multipath routing algorithm is shown in Table 2, the performance of multipath fault tolerance and HPAMR fault tolerance is shown in Figure 3.

\subsection{Transmission Speed}

Second parameter is transmission speed in the sensor network, multipath routing and sensor node communication everything based on single node communication but proposed model is based on clustering technique in the WSN. All the nodes transfer the data to $\mathrm{CH}$ and $\mathrm{CH}$ is forward the information to neighbor cluster head so the

Table 1. Simulation parameter.

\begin{tabular}{cc}
\hline Parameter & Count \\
\hline Simulator & NS 3.23 \\
Operating System & Ubuntu 14.04 \\
Number of Clusters & 20 \\
Data Packet Size & $4 \mathrm{~kb}$ \\
Simulation Duration & 10 Minutes \\
\hline
\end{tabular}

Table 2. Comparison of multipath Fault tolerance and HPAMR Fault tolerance.

\begin{tabular}{|c|c|c|}
\hline No. of Clusters Algorithm & $\begin{array}{l}\text { Multipath Routing } \\
\text { (Fault tolerance in percentage) }\end{array}$ & $\begin{array}{c}\text { HPAMR } \\
\text { (Fault tolerance in percentage) }\end{array}$ \\
\hline 10 & 68 & 72 \\
\hline 20 & 72.7 & 77 \\
\hline 30 & 75 & 80.5 \\
\hline 50 & 78 & 83 \\
\hline 100 & 83 & 85 \\
\hline
\end{tabular}




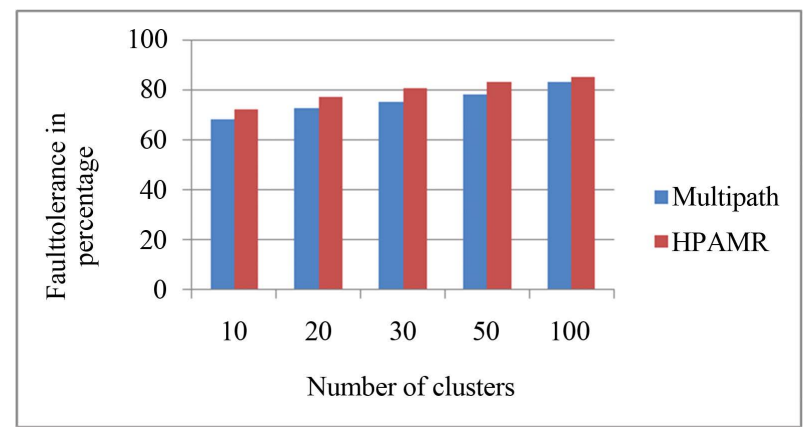

Figure 3. Performance of multipath fault tolerance and HPAMR fault tolerance.

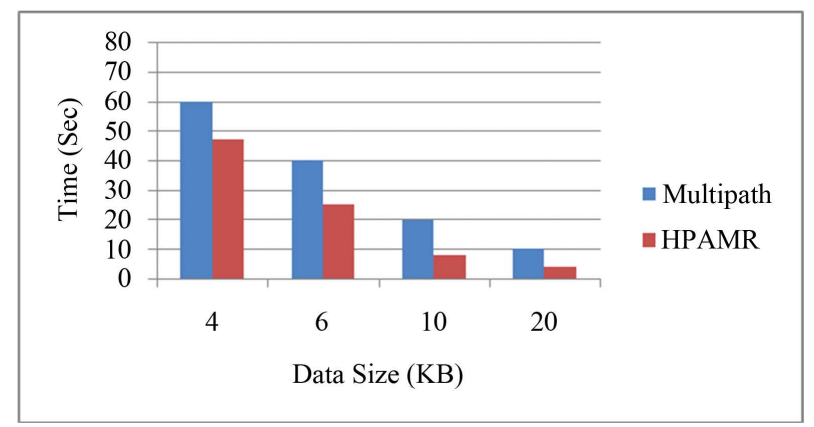

Figure 4. Performance of Multipath Data Packet Speed and HPAMR.

Table 3. Comparison of multipath data packet speed and HPAMR.

\begin{tabular}{|c|c|c|c|}
\hline No of Clusters Algorithm & Data Size & $\begin{array}{l}\text { Multipath Routing } \\
\text { Time in sec }\end{array}$ & $\begin{array}{c}\text { HPAMR } \\
\text { Time in sec }\end{array}$ \\
\hline 10 & $4 \mathrm{~KB}$ & $60 \mathrm{sec}$ & $47 \mathrm{sec}$ \\
\hline 20 & $6 \mathrm{~KB}$ & $40 \mathrm{sec}$ & $25 \mathrm{sec}$ \\
\hline 30 & $10 \mathrm{~KB}$ & $20 \mathrm{sec}$ & $8 \mathrm{sec}$ \\
\hline 50 & $20 \mathrm{~KB}$ & $10 \mathrm{sec}$ & $4 \mathrm{sec}$ \\
\hline
\end{tabular}

process is very speed because $\mathrm{CH}$ is act a leader in a cluster it collect the information from other nodes participating in the cluster. Another thing is if congestion or path failure occur in a network the proposed model check the available cluster and transfer the data based on our discussion. Table 3 shows the comparison of multipath speed and HPAMR speed in WSCE. Performance of multipath data packet speed and HPAMR is shown in Figure 4.

\section{Conclusion}

The proposed model of this paper deals with high-performance adaptive multipath routing algorithm implemented in wireless sensor cloud environment by applying with CPEGASIS protocol. The proposed algorithm increases the fault tolerance and data speed in the wireless network, where the simulation data transmission time is reduced by our proposed HPAMR algorithm. The algorithm is used to transfer the data source (i.e. sensor cluster gateway) to cloud environment without any data loss in the network. Finally, the result of the proposed methodology is used to increase the fault tolerance, data speed and to reduce the data transmission time in the wireless sensor cloud environment.

\section{References}

[1] Somani, A.K. and Vaidya, N.H. (1997) Understanding Fault Tolerance and Reliability. 
[2] Kannan, B. and Parker, L.E. (2006) Fault-Tolerance Based Metrics for Evaluating System Performance in Multi-Robot Teams. Proceedings of Performance Metrics for Intelligent Systems Workshop.

[3] Tsai, J. and Moors, T. A Review of Multipath Routing Protocols: From Wireless Ad Hoc to Mesh Networks. National ICT Australia (NICTA) 1/University of New South Wales, Australia.

[4] Al-Karaki, J.N. and Kamal, A.E. (2004) Routing Techniques in Wireless Sensor Networks: A Survey. IEEE Wireless Communications, 11, 6-28.

[5] Aslam, M.S., Rea, S. and Pesch, D. (2012) Service Provisioning for the WSN Cloud. IEEE Fifth International Conference on Cloud Computing.

[6] Narasimha Datta, N. and Gopinath, K. (2006) A Survey of Routing Algorithms for Wireless Sensor Networks. Journal of the Indian Institute of Science.

[7] Saranya, B. and Subha, R. (2014) Performance and Energy Efficient of Comparing Routing Algorithms. International Journal of Advanced Research in Computer Science and Software Engineering, 4, No. 7.

[8] Rajasekaran, K. and Balasubramanian, K. (2016) Energy Conscious Based Multipath Routing Algorithm in WSN. I. J. Computer Network and Information Security, 1, 27-34.

[9] Matam, R. and Tripathy, S. (2016) Secure Multicast Routing Algorithm for Wireless Mesh Networks. Journal of Computer Networks and Communications, 2016.

[10] Nikolidakis, S.A., Kandris, D., Vergados, D.D. and Douligeris, C. (2013) Energy Efficient Routing in Wireless Sensor Networks through Balanced Clustering. Algorithms, 6, 29-42.

[11] Liu, R.L., et al. (2015) Balanced Traffic Routing: Design, Implementation, and Evaluation. Ad Hoc Networks, 37, 1428.

[12] Udhayakumar, S., Selvan, L.T., Umanandhini, D., RajKumar, U. and Dhinakaran, K. (2012) Power Aware Zone Based Routing in a Pervasive Irrigation Management System. 2012 Third International Conference on Computing Communication \& Networking Technologies, Tamilnadu, 26-28 July 2012.

[13] Najjar, W. and Gaudiot, J.-L. (1990) Network Resilience: A Measure of Network Fault Tolerance. IEEE Transactions on Computers, 39. No. 2.

\section{Submit or recommend next manuscript to SCIRP and we will provide best service for you:}

Accepting pre-submission inquiries through Email, Facebook, LinkedIn, Twitter, etc.

A wide selection of journals (inclusive of 9 subjects, more than 200 journals)

Providing 24-hour high-quality service

User-friendly online submission system

Fair and swift peer-review system

Efficient typesetting and proofreading procedure

Display of the result of downloads and visits, as well as the number of cited articles

Maximum dissemination of your research work

Submit your manuscript at: http://papersubmission.scirp.org/ 